\title{
Genome sequence of the stramenopile Blastocystis, a human anaerobic parasite
}

France Denoeud ${ }^{1 \dagger}$, Michaël Roussel ${ }^{2,3 \dagger}$, Benjamin Noel ${ }^{1}$, Ivan Wawrzyniakk ${ }^{2,3}$, Corinne Da Silva', Marie Diogon ${ }^{2,3}$, Eric Viscogliosi ${ }^{4,5,6,7}$, Céline Brochier-Armanet ${ }^{8,9}$, Arnaud Couloux ${ }^{1}$, Julie Poulain ${ }^{1}$, Béatrice Segurens ${ }^{1}$, Véronique Anthouard ${ }^{1}$, Catherine Texier ${ }^{2,3}$, Nicolas Blot ${ }^{2,3}$, Philippe Poirier ${ }^{2,3}$, Geok Choo Ng ${ }^{10}$, Kevin SW Tan ${ }^{10}$, François Artiguenave ${ }^{1}$, Olivier Jaillon ${ }^{1}$, Jean-Marc Aury ${ }^{1}$, Frédéric Delbac ${ }^{2,3}$, Patrick Wincker ${ }^{1 *}$, Christian P Vivarès ${ }^{2,3^{*}}$ and Hicham El Alaoui ${ }^{2,3^{*}}$

\begin{abstract}
Background: Blastocystis is a highly prevalent anaerobic eukaryotic parasite of humans and animals that is associated with various gastrointestinal and extraintestinal disorders. Epidemiological studies have identified different subtypes but no one subtype has been definitively correlated with disease.

Results: Here we report the $18.8 \mathrm{Mb}$ genome sequence of a Blastocystis subtype 7 isolate, which is the smallest stramenopile genome sequenced to date. The genome is highly compact and contains intriguing rearrangements. Comparisons with other available stramenopile genomes (plant pathogenic oomycete and diatom genomes) revealed effector proteins potentially involved in the adaptation to the intestinal environment, which were likely acquired via horizontal gene transfer. Moreover, Blastocystis living in anaerobic conditions harbors mitochondria-like organelles. An incomplete oxidative phosphorylation chain, a partial Krebs cycle, amino acid and fatty acid metabolisms and an iron-sulfur cluster assembly are all predicted to occur in these organelles. Predicted secretory proteins possess putative activities that may alter host physiology, such as proteases, protease-inhibitors, immunophilins and glycosyltransferases. This parasite also possesses the enzymatic machinery to tolerate oxidative bursts resulting from its own metabolism or induced by the host immune system.

Conclusions: This study provides insights into the genome architecture of this unusual stramenopile. It also proposes candidate genes with which to study the physiopathology of this parasite and thus may lead to further investigations into Blastocystis-host interactions.
\end{abstract}

\section{Background}

Blastocystis sp. is one of the most frequent unicellular eukaryotes found in the intestinal tract of humans and various animals [1]. This anaerobic parasite was first described by Alexeieff at the beginning of the 20th century [2]. For a long time, the taxonomy of Blastocystis was controversial. Despite the application of molecular phylogenetic approaches, it was only recently that

\footnotetext{
*Correspondence: pwincker@genoscope.cns.fr; christian.vivares@univbpclermont.fr; hicham.el_alaoui@univ-bpclermont.fr

+ Contributed equally

${ }^{1}$ Genoscope (CEA) and CNRS UMR 8030, Université d'Evry, 2 rue Gaston Crémieux, 91057 Evry, France

${ }^{2}$ Clermont Université, Université Blaise Pascal, Laboratoire Microorganismes: Génome et Environnement, BP 10448, F-63000 Clermont-Ferrand, France
} Full list of author information is available at the end of the article
Blastocystis sp. was unambiguously classified within the stramenopiles [3-5]. This eukaryotic major lineage, also called Heterokonta, encompasses very diverse organisms (unicellular or multicellular, heterotrophic or photosynthetic) such as slime nets, diatoms, water moulds and brown algae [6]. One important characteristic of stramenopiles is the presence during the life cycle of a stage with at least one flagellum permitting motility. It is important to note that Blastocystis sp. does not possess any flagellum and is the only stramenopile known to cause infections in humans [4]. For the organism isolated from human fecal material, Brumpt suggested the name Blastocystis hominis [7]. However, as the species $B$. hominis is difficult to establish, we use the term 'Blastocystis sp.' to designate any subtype observed in

\section{Biomed Central}


humans. Blastocystis sp. is the most frequent protozoa reported in human fecal samples [8], with a worldwide distribution [9-13] and a prevalence ranging between 30 and $60 \%$ in some developing countries [1]. In addition, infection with Blastocystis sp. appears to be common and more severe in immunocompromised or hemophilic patients $[9,14,15]$. The presence of Blastocystis representatives has also been reported in a variety of mammals, birds, reptiles, and even insects [16-18]. Blastocystis sp. exhibits extensive genetic diversity, and on the basis of molecular analysis of the small subunit RNA gene, ten distinct subtypes (ST1 to ST10) have been identified from primates (including humans), other mammals and birds [17]. Some arguments support zoonotic transmission to humans, including the high prevalence of ST1 to ST3 in humans and other mammals [17] and the experimental transmission of different human genotypes to chickens, rats and mice $[19,20]$.

The life cycle of Blastocystis sp. remains elusive, although different morphological forms have been described, including vacuolar, granular, amoeboid and cysts. Recently, Tan [1] suggested a life cycle with the cyst as the infectious stage. After ingestion of cysts, the parasite may undergo excystation in the gastrointestinal tract and may develop into a vacuolar form that divides by binary fission. The following stage could be either the amoeboid form or the granular form. Then, encystation may occur during passage along the colon before cyst excretion in the feces. Therefore, Blastocystis sp. lives in oxygen-poor environments and is characterized by the presence of some double-membrane surroundedorganelles showing elongate, branched, and hooked cristae [21] called mitochondria-like organelles (MLOs) [22]. These cellular compartments contain a circular DNA molecule and have metabolic properties of both aerobic and anaerobic mitochondria [23,24].

Blastocystis sp. has been reported as a parasite causing gastro- and extra-intestinal diseases with additional persistent rashes, but a clear link of subtypes to the symptomatology is not well established [11]. Other studies have shown that the parasite can be associated with irritable bowel syndrome $[20,25]$ or inflammatory bowel disease [26]. Thus, the pathogenic role of Blastocystis sp. as the primary cause of enteric symptoms is dubious. Therefore, it is important to search for other molecular markers for an epidemiologically integrated study [17]. Here we report the complete genome sequence of a subtype 7 isolate from a Singaporean patient [GenBank: CABX01000000]. Its comparison with the two other available stramenopile genome sequences (that is, Phytophthora sojae, a plant pathogenic oomycete, and Thalassiosira pseudonana, a free diatom) allows us to highlight some genome-specific features of Blastocystis to understand how this parasite evolved within environmental constraints, but also provides a better knowledge of its metabolic and physiological capacities, such as the functioning and the role of MLOs and the arsenal produced to interact or to counter immune defense systems of its host.

\section{Results and discussion}

\section{General features of the Blastocystis genome}

The genome of a Blastocystis subtype 7 was resolved by pulsed-field gel electrophoresis, and 15 chromosomic bands have been characterized. The final assembled sequence is distributed in 54 scaffolds and the deduced genome is $18.8 \mathrm{Mb}$ in size (16.5-fold sequence coverage), which is much smaller than plant parasite stramenopiles (Phytophthora infestans, $240 \mathrm{Mb}$; P. sojae, $95 \mathrm{Mb}$; Phytophthora ramorum, $65 \mathrm{Mb}$ ) and also smaller than free stramenopiles (Phaeodactylum tricornutum, 27.4 Mb; T. pseudonana, $34.5 \mathrm{Mb}$ ). The reference annotation of the Blastocystis subtype 7 genome contains 6,020 genes, covering about $42 \%$ of the genome (Table 1 ). The average number of exons per gene is 4.6 for multiexonic genes and 929 genes are monoexonic. Compaction in this parasite genome is reflected by the short length of the intergenic regions $(1,801 \mathrm{bp})$, the relatively low repeat coverage (25\%) and, more strikingly, by the very short size of introns, with a sharp length distribution of around 32 nucleotides (Figure S1 in Additional file 1). A total of 38 rDNA units organized in transcriptional units, including a small subunit rRNA gene, a 5.8S rRNA gene, and a large subunit rRNA gene in a 5'-3' orientation, have been detected in the genome. The sizes of the small subunit, the large subunit and the $5.8 \mathrm{~S}$ rRNA gene are $1.8 \mathrm{~kb}$, $2.45 \mathrm{~kb}$ and $0.44 \mathrm{~kb}$, respectively. Some units are tandemly duplicated, up to four copies on scaffold 18, and some may also be localized in subtelomeric regions, as revealed by a co-mapping of telomeric sequences and rDNA subunits at scaffold 6 and 9 extremities. These two scaffolds could correspond to entire chromosomes. Due to the sequencing method, some units are incomplete (either truncated or lacking genes). The alignment of 20 complete small subunit rRNA genes shows polymorphism between copies, which is also the case for 29 large subunit rRNA gene copies.

The number of genes in Blastocystis $(6,020)$ is reduced in comparison with other stramenopiles (P. infestans, 17,797; P. sojae, 19,027; P. ramorum, 15,743; P. tricornutum, 10,402; T. pseudonana, 11,776). Surprisingly, a large portion of genes were probably duplicated since 404 clusters of paralogous protein-coding genes were identified, containing 1,141 genes, that is, $19 \%$ of Blastocystis genes (see Material and methods). Excluding the large multigenic families (up to 32 genes with a histone-fold domain and 20 genes with a $4 \mathrm{Fe}-4 \mathrm{~S}$ ferredoxin domain), most of the duplicated genes are present in only two copies (Figure S2 in 
Table 1 General features of Blastocystis sp. subtype 7

\begin{tabular}{lccccc}
\hline & Number & Mean length & Median length & Total length $(\mathbf{M b})$ & Percentage of genome (18.8 Mb) \\
\hline Genes & 6,020 & 1,299 & 1,397 & 7.82 & $42 \%$ \\
Exons & 24,580 & 280 & 150 & 6.88 & $37 \%$ \\
Introns & 18,560 & 50.5 & 31 & 0.94 & $5 \%$ \\
Intergenic & - & 1,801 & 4,092 & 10.9 & $58 \%$ \\
Repeats & 2,730 & 1,747 & 2,862 & 4.8 & $25 \%$ \\
\hline
\end{tabular}

Additional file 1). As described in other organisms [27,28], the duplicated genes are more conserved than single copy genes in Blastocystis sp. Indeed, they have more orthologs (defined as best reciprocal hit (BRH); see Materials and methods) and display higher similarities with their orthologs (Figure S3 in Additional file 1). They also tend to display higher expression levels than single copy genes (Figure S4 in Additional file 1).

We investigated whether these gene duplications could have arisen from a whole genome duplication (WGD) or smaller scale segmental duplications. WGD, the duplication of the entire genome by polyploidization, has been shown to have played a key role in the evolutionary history of several animal and plant lineages [27,29-31]. Segmental duplications occur continually by several mechanisms that can duplicate parts of genes, entire genes, or several adjacent genes. These mechanisms include unequal crossing over, or gene conversion, and tandem duplication [32-34]. We were able to identify 320 blocks of duplicated genes, that is, paralogous segments of several adjacent genes (see Materials and methods), some of which are very large (up to $100 \mathrm{~kb}$ ), suggesting a WGD. These blocks cover about $39 \%$ of the genome ( 7.3 out of $18.8 \mathrm{Mb}$ ) representing $38 \%$ (5.15 out of $13.65 \mathrm{Mb}$ ) of the unrepeated fraction of the genome. As shown in Figure 1, each scaffold is a mosaic of blocks of homology with several other scaffolds: scaffolds cannot be grouped by pairs as would be expected from a recent WGD. Additionally, some segments are present in more than two copies in the genome (they appear in black in Figure 1), suggesting that segmental duplications are likely to have played a role in the current duplication pattern. However, the duplicated blocks are not often on the same scaffold, nor in tandem, which rules out the tandem duplication model. The comparison of paralogous copies shows surprisingly high nucleic acid identity rates: on average, $99 \%$ in coding regions, $98.4 \%$ in untranslated regions, and $97.8 \%$ in introns and intergenic regions. Interestingly, those values are homogeneous among all paralogous blocks, suggesting that all blocks were duplicated at the same time.

Two hypotheses could explain the origin of these duplicated blocks. First, the duplicates may have arisen from a whole genome duplication that took place recently (since the copies are still very similar) and was followed by rapid genome rearrangements and losses of gene copies. The high homology between gene copies could also result from a high rate of homogenization through gene conversion driven by the high frequency of rearrangements. The frequent rearrangements in the Blastocystis lineage are probably also the reason why no extensive synteny could be detected between Blastocystis sp. and other stramenopiles. Second, the duplicates could also have occurred through segmental duplications (favored by the high rate of rearrangements), although the relatively uniform divergence between copies is more symptomatic of a single event and would imply a burst of segmental duplications during a short period or a very high rate of homogenization by recombination. The intriguing pattern of gene duplications, likely caused by the high rate of rearrangements in the Blastocystis genome, makes it impossible to determine which scenario is the most likely. It could be interesting to sequence other subtypes to determine whether the high rate of recombination (loss of synteny) and the pattern of duplications observed in subtype 7 is a common feature within this lineage.

\section{Endosymbiotic and horizontal gene transfers in Blastocystis sp}

Phylogenetic analyses revealed two genes of possible cyanobacterial origin in the genome of Blastocystis, those encoding phosphoglycerate kinase [GenBank: CBK20833] and 6-phosphogluconate dehydrogenase [GenBank:CBK22626] (Figure S5 in Additional file 1). It is important to notice that 6-phosphogluconate dehydrogenase-encoding genes have been identified in non-photosynthetic protists such as Heterolobosea (not shown). This was interpreted as secondary horizontal gene transfer (HGT) from photosynthetic eukaryotes to Heterolobosea [35,36].

The presence of plastids in various photosynthetic stramenopile lineages (for example, diatoms, chrysophytes, raphidophytes) was interpreted as a secondary endosymbiosis that occurred between a red algae and the ancestor of these groups. By contrast, the evolutionary meaning of the lack of plastids in some heterotrophic stramenopile lineages (for example, oomycetes, bicosoesids) is still under discussion: does it indicate secondary losses of the 


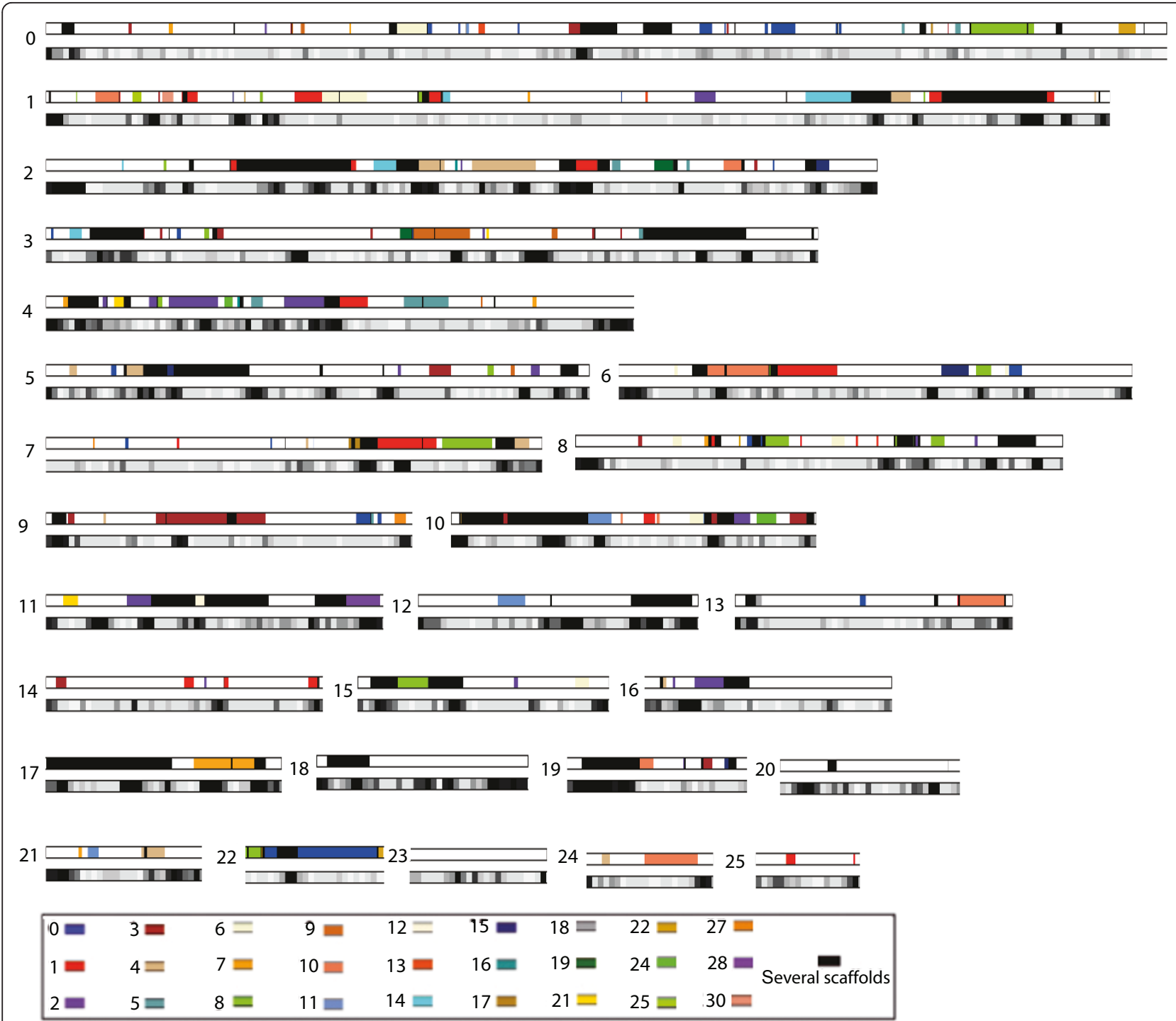

Figure 1 Blocks of duplicated genes in the Blastocystis sp. genome. For each scaffold (from 0 to 25), the duplicated blocks are displayed with colors corresponding to the scaffolds where the paralogous blocks are located (on scaffolds 0 to 19, 21, 22, 24, 25, 27, 28, 30). Below each scaffold, the repeat density is displayed as a grey scale: $0 \%$ (white) to 100\% (black) repeats in 10-kb windows.

plastid acquired by the ancestor of all stramenopiles? Or does it reflect the fact that the secondary endosymbiosis at the origin of stramenopile plastids did not occur in their common ancestor but after the divergence of heterotrophic lineages [37]? The presence of genes of cyanobacterial origin in Blastocystis supports the first hypothesis even if we can not rule out possible recent acquisitions of genes of chloroplastic origin from photosynthetic eukaryotes as in the case of Heterolobosea.

HGT is important in evolution as an adaptive mechanism of microbial eukaryotes to environmental conditions $[38,39]$ and is known to play an important role in stramenopiles. For instance, iron is a limiting nutrient in surface waters for diatoms. Therefore, the likely acquisition of ferritin by HGT from bacteria has permitted some species to acquire this nutrient from the environment [40]. This is also the case for the diatom Phaeodactylum, in which nitrogen metabolism, cell wall silification, DNA replication, genome repair and recombination processes have been shaped by HGT [40,41]. HGT seems also to play an important role in oomycetes since it may be involved in osmotrophy. Genes involved in absorbing products of degradation of complex nutrients were predicted to be candidates for fungi-to-oomycete HGT [42]. By analyzing the set of predicted genes in Blastocystis sp. that are homologous to bacterial or archaeal genes, we identified 133 candidates for HGT (Table S3 in Additional file 2). In most cases, our phylogenetic analyses 
confirm the bacterial origin of these genes even if they were not sufficiently resolved to allow the precise identification of the donor, suggesting that these HGT events were ancient and/or that the corresponding genes are rapidly evolving in the genome of Blastocystis sp. Interestingly, in a few cases, even when the transferred gene is of bacterial origin, the Blastocystis sp. copy is closely related to homologues found in pathogenic and/or anaerobic eukaryotes, suggesting that HGT between eukaryotes has played a key role in these organisms too (Figure S6 in Additional file 1).

Some of the genes that originated from HGT possess functions that lead to a better understanding of how this lineage emerged. Three are homologous to the bacterial major facilitator transporter (MFS_1), the first two being nearly identical, and therefore resulting from a recent gene duplication event. MFS proteins form a large and diverse group of secondary transporters, which facilitate the transport across membranes of a variety of substrates, including ions, sugar phosphates, drugs, neurotransmitters, nucleosides, amino acids and peptides [43]. Two Blastocystis MFS genes have closely related homologues in some pathogenic eukaryotes like the Alveolata Perkinsus marinus or fungi such as Gibberella zeae and Verticillium albo atrum, suggesting an acquisition from bacteria followed by HGT between these eukaryotes (Figure S6f in Additional file 1). However, the phylogeny resolution is too low to precisely identify the bacterial donor of these genes. The presence of MSF proteins in Blastocystis sp. may confer the ability to absorb nutrients from the environment to this parasite, particularly in the intestinal lumen or when attacking host tissues. We have also found different HGT genes harboring alcohol deshydrogenase, short-chain dehydrogenase and oxidoreductase domains (Table S3 in Additional file 2) that may be involved in specific fermentations that remain to be characterized. Some of them are closely related to homologues found in anaerobic eukaryotes like Trichomonas vaginalis and Entamoeba histolytica (Figure S6b in Additional file 1) or in the bacteria Legionella pneumophila or Parachlamydia acanthamoebae, which infect or are associated with amoeba $[44,45]$. These enzymes may increase the range of Blastocystis sp. metabolic abilities to produce energy in anaerobic environments, as has been observed in Giardia lamblia and E. histolytica $[46,47]$.

Several genes acquired by HGT may participate in the adhesion of the parasite to the host tissues. Indeed, 26 genes (Table S3 in Additional file 2) encode proteins containing the IPR008009 domain, which is often associated with immunoglobulin domains, a conserved core region of an approximately 90 -residue repeat found in several hemagglutinins and other cell surface proteins. Among these 26 Blastocystis sp. proteins, some also contain the IPR015919 domain, which characterizes cadherins, a family of adhesion molecules that mediate $\mathrm{Ca}^{2+}$-dependent cell-cell adhesion. Homologous genes are also found in some beta-Proteobacteria or Acidobacteria, but the sequences are very divergent and our phylogenetic analysis did not, therefore, allow firm identification of the bacterial donor. Some hydrolaseencoding genes could also result from the transfer from bacteria to Blastocystis sp. One of them possesses an esterase-lipase (IPR013094) domain (Table S3 in Additional file 2) and may participate in the degradation of host tissue during infection. The closest homologues of this gene are found in the fungus Botryotinia fuckeliana, in Firmicutes and Actinobacteria (Figure S6d in Additional file 1).

Overall, these HGT genes may have allowed flexibility in genome expression, enabling the successful adaptation of Blastocystis sp. to digestive environments through genes encoding proteins that could be involved in osmotrophy (MFS), energy metabolism (dehydrogenases) and adhesion.

\section{Circular genome, predicted proteome and metabolic pathways of the MLOs}

Although it lives in anaerobic or microaerophilic conditions, Blastocystis sp. harbors MLOs that present both mitochondrial and hydrogenosomal features [24]. We recently reported that Blastocystis sp. MLOs contain a circular genome, including genes encoding 10 of the 20 complex I subunits, but they lack all genes encoding cytochromes, cytochrome oxidases and ATP synthase subunits [24], unlike mitochondrial DNA from other sequenced stramenopiles, such as Phytophthora sp. [48]. The MLO genome of the Blastocystis subtype 7 is a circular molecule 29,270 bp in size. Two other MLO genomes were then sequenced from isolates belonging to other subtypes [49]: a subtype 1, represented by Blastocystis Nand II, with a 27,719 bp genome; and a subtype 4, represented by Blastocystis DMP/02-328, with a 28,382 bp genome. In addition to sequence conservation, these three genomes have many similarities. Their $\mathrm{A}+\mathrm{T}$ content is around $80 \%$, their gene density is higher than $95 \%$ and all three encompass 45 genes: 27 ORFs, 16 tRNAs and 2 rRNA genes. The ORFs consist of $\mathrm{NADH}$ subunits, ribosomal proteins and proteins with no similarity in the databases. The synteny between the three MLO genomes is highly conserved: gene order is strictly the same among the three genomes $[24,49]$.

Through the analysis of a Blastocystis EST database, Stechmann et al. [23] have identified 110 potential proteins associated with mitochondrial pathways, such as the oxidative phosphorylation chain, tricarboxylic acid (TCA) cycle, Fe/S cluster assembly, and amino acid and fatty acid metabolisms. Nonetheless, approximately half of these proteins have an incomplete amino terminus 
due to EST data, making it difficult to confirm mitochondrial import by algorithms. To clarify the metabolic characteristics of these puzzling organelles, we used data from the whole genome sequence in order to establish the in silico proteome of Blastocystis MLOs. For this purpose, a computational approach based on two different prediction algorithms (MitoProt and MitoPred) for mitochondrial-import proteins was chosen (see Materials and methods for more details). This approach predicted 365 MLO proteins (Table S6 in Additional file 3) whereas Stechmann et al. [23] predicted only 110 proteins. Among these 365 proteins, 299 were predicted to have an amino-terminal extension involved in mitochondrial import, suggesting that an alternative system might exist for the 66 remaining proteins. Of the 299 proteins, 41 remain as 'hypothetical protein' with unknown function and 31 have no homologues in public databases, which raises the question of the existence of undiscovered metabolic processes within these intriguing organelles (Table S6 in Additional file 3). The other proteins are involved in classical mitochondrial core functions, such as oxidative phosphorylation, amino acid metabolism, fatty acid oxidation, iron-sulfur cluster assembly, and mitochondrial import system. Several proteins involved in the translocase of the outer mitochondrial membrane (TOM complex), the translocase of the inner membrane (TIM complex), and the presequence translocase-associated motor (PAM complex), which perform protein transport into the matrix, were identified. Interestingly, the two essential subunits of the mitochondrial processing peptidase heterodimer (MPP $\alpha / \beta)$, essential for the cleavage of the targeting peptide, were also found [50].

Our analyses revealed that MLOs probably have three ways to make acetyl-CoA from pyruvate, supported by the presence of the pyruvate dehydrogenase complex, pyruvate:ferredoxin oxidoreductase and pyruvate:NADP ${ }^{+}$ oxidoreductase (an amino-terminal pyruvate:ferredoxin oxidoreductase domain fused to a carboxy-terminal $\mathrm{NADPH}$-cytochrome P450 reductase domain) (Figure 2). Euglena gracilis mitochondria include this feature, which provides adaptability to various oxygen levels [51], and this might be to a lesser extent the case for Blastocystis sp. We have also identified the 20 subunits of the Blastocystis sp. MLO complex I (ten are encoded by the MLO genome and ten by nuclear genes). The four nuclear-encoded subunits of the mitochondrial respiratory chain complex II were detected and this complex could function in two ways (via succinate dehydrogenase or fumarate reductase) [52]. We did not identify any genes encoding complexes III and IV subunits or ATP synthase. However, we have found components of the TCA cycle, which was shown to be involved with complex II (fumarate reductase) in fumarate respiration in parasitic helminths [52]. Interestingly, we identified a gene encoding a terminal oxidase, called alternative oxidase (AOX), which could be the terminal electron acceptor of complexes I and II (Figure 2), allowing adaptation to oxygen stress and maintaining the NADH/ NAD balance, as has been suggested for Cryptosporidium parvum $[53,54]$. These data raise questions about the electron acceptor when complex II has succinate dehydrogenase or fumarate reductase activity, the quinone used in this process and the role of the proton gradient.

We also revealed proteins that can be grouped into essential mitochondrial pathways, like the $\mathrm{Fe} / \mathrm{S}$ cluster assembly. More precisely, we have identified 11 enzymes (6 of which have predicted mitochondrial import signals), composing the iron-sulfur cluster system responsible for the assembly of mitochondrial $\mathrm{Fe} / \mathrm{S}$ proteins [55], such as the cysteine desulfurase Nfs1, the scaffold protein Isu1, frataxin, and the P-loop NTPase Ind1, which is required for the assembly of complex I (Figure 2). We also highlighted some proteins involved in mitochondrial fatty acid synthesis type II [56], beta oxidation of fatty acids and amino acid metabolism (Table S6 in Additional file 3).

Taken together, our data confirm the mitochondrial nature of the Blastocystis sp. MLO. The oxygen-poor environment may have driven the selection of these unique organelles, which seemingly represent an intermediate situation between anaerobic mitochondria and hydrogenosomes, arguing for multiple situations arising during organelle evolution. It remains now to describe the metabolism occurring in these unusual organelles more precisely.

\section{Secretome and virulence factors}

The persistence of Blastocystis sp. in the host may be due, to some extent, to its ability to override the response of the immune system and to adhere and survive within the intestinal tissue. Manipulation of the host might be facilitated by molecules released at the interface between the host and the parasite [57]. Accordingly, the study of the predicted secretome of Blastocystis sp. is of particular interest. With SIGNALP 3.0, 307 proteins were predicted to be secretory, of which 46 had no sequence similarity in the public $\mathrm{nr}$ databases. By sequence homology, 170 proteins that could play a role in host-parasite relationships were selected and submitted to PSORTII for extracellular location. Finally, 75 putative secreted proteins have been classified by putative functions, some of which may have a direct connection with pathogenicity (proteases, hexose digestion enzymes, lectins, glycosyltransferases and protease inhibitors; Table S4 in Additional file 2).

Blastocystis can secrete members of the immunophilin family, characterized by peptidyl-propyl cis-trans 


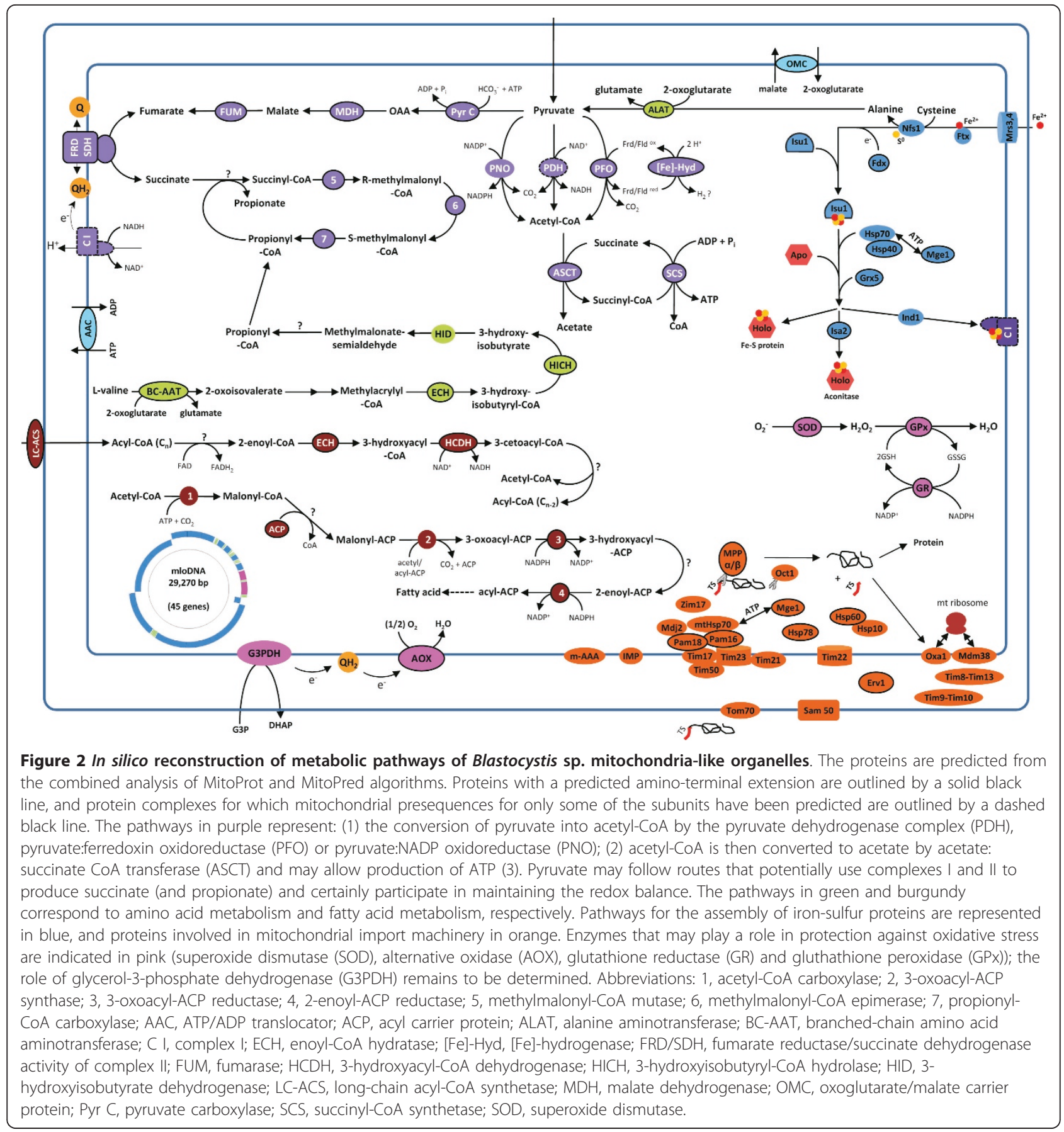

isomerase activity and disulfide isomerases (Figure 3; Table S4 in Additional file 2). These proteins have key roles in protein folding, but it has also been established that they can have moonlighting functions. In bacteria, they have evolved adhesive properties for the host [58] but they can also modulate host leukocyte function and induce cellular apoptosis [59]. A cyclophilin-like protein from the protozoan parasite Toxoplasma gondii is directly involved in host-parasite crosstalk, as it can modulate protective Th1 responses through its binding to the chemokine receptor CCR5 [60]. It is unclear what role these proteins play in Blastocystis sp., but this illustrates a range of functions for cell stress proteins in host-pathogen interactions.

Sugar-binding proteins have an important role through a conserved carbohydrate-recognition domain that could interact with host cell receptors. Such proteins have been characterized in other parasites [61] and 


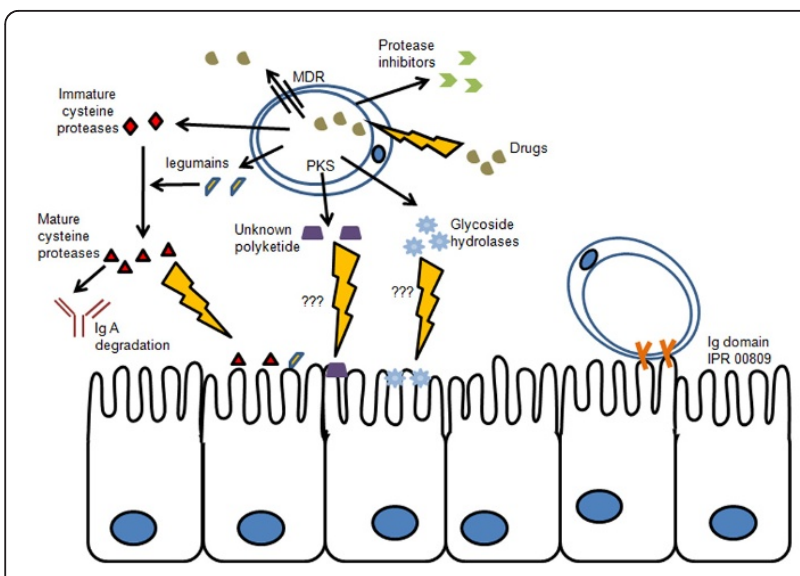

Figure 3 Secretory proteins and virulence factors identified in the Blastocystis sp. subtype 7 potentially involved in host interaction. Blastocystis sp. may release cysteine proteases, which could be processed by legumain. These proteases may attack intestinal epithelium together with other hydrolases, such as glysoside hydrolases. Protease inhibitors, some of which have been predicted to be secreted, could act on host proteases (digestive enzymes or proteases involved in the immune response). Some as yet uncharacterized secondary metabolites produced by polyketide synthase (PKS) identified in the genome could also participate in host intestinal symptoms. Adhesive candidate proteins (proteins with an immunoglobulin Ig domain) have been found. Finally, drugresistant isolates of the parasite could be explained by the presence of multidrug resitance (MDR) proteins. Lightning bolts indicate potential toxic effects.

it is interesting to note that some sugar-binding proteins are able to inhibit Th1- and Th2-mediated inflammation $[62,63]$. Moreover, some specific sugar-binding proteins are also able to suppress regulatory $\mathrm{T}$ cells [64]. The binding of these proteins is dependent on their specific sugar motifs, which can be added to $\mathrm{N}$ - or O-linked glycans by glycosyltransferases. One carbohydrate-binding protein and eight glycosyltransferases (Table S4 in Additional file 2) have been predicted to be secreted. All these enzymes could allow cross-linking of Blastocystis sp. sugar-binding proteins to host cell receptors.

The parasite likely uses hydrolases to attack host tissues. Fucosidase, hexosaminidase and polygalacturonase have been identified in the predicted secretome and may participate in this process by degrading host glycoproteins (Figure 3; Table S4 in Additional file 2). Proteases have been proposed to be involved in diverse processes, such as host cell invasion, excystation, metabolism, cytoadherence or other virulence functions. A correlation between a high level of protease activity and the virulence of the intestinal parasite E. histolytica was proven by McKerrow et al. [65]. Indeed, cysteine proteases degrade extracellular matrix proteins, cleave immunoglobulin A and G, and are thought to be responsible for the cytopathic effect of different pathogens against in vitro cultured cells [66]. Interestingly, Blastocystis sp. proteolytic enzymes are also able to degrade human secretory immunoglobulin A [67]. All the major classes of proteolytic enzymes were identified in the genome data, including serine, aspartic, and cysteine proteases and metalloproteases. Among the 66 proteases identified, 18 are predicted to be secreted by the parasite (Table S4 in Additional file 2). Within the protease family, cysteine protease-encoding genes are the most represented in Blastocystis sp. genome and $96 \%$ of the proteins encoded by these genes are predicted to be secreted. Among the cysteine proteases we have found five legumains and eight cathepsins; three cathepsins B contain the IPR015643 domain, which is only present in Blastocystis sp. compared to the other stramenopiles. The IPR015643 domain corresponds to the peptidase $\mathrm{C} 1$ cathepsin $\mathrm{B}$ domain and has a cysteine type peptidase activity, which was also found in pathogenic protozoa (Leishmania sp. and Trypanosoma sp.) [66]. Cysteine proteases are usually secreted in their inactive form and must be matured, having a prosegment that prevents hydrolysis during protease trafficking and storage. This maturation might result from the activity of the same protease or another, such as asparaginyl endopeptidase (also called legumain) [68]. This endopeptidase cleaves peptide bonds carboxy-terminal to asparagine residues, and may be involved in processing and activating both cathepsins $\mathrm{L}$ and $\mathrm{B}$. Legumains have been predicted in the secretome of Blastocystis sp. (Table S4 in Additional file 2) and could be involved in protease processing (Figure 3). As an alternative role, secreted Blastocystis sp. legumains could also participate with other effectors in the alteration of the host intestine [69]. Indeed, it has been shown that legumain can degrade fibronectin, an extracellular matrix glycoprotein [70].

Genes coding for protease inhibitors are also present in the Blastocystis sp. genome, and some are predicted to be secreted. Release of protease inhibitors may weaken the host response as described in nematodes [71]. Blastocystis sp. encodes three protease inhibitors: cystatin, type1proteinase inhibitor and endopeptidase inhibitor-like protein (Table S4 in Additional file 2). Type1-proteinase inhibitor is similar to chymotrypsin inhibitor, which is known to inactivate intestinal digestive enzymes (trypsin and chymotrypsin) as in Ascaris suum [72], thus protecting the parasite against non-specific digestive defenses. Cystatin, also called stefin, was described in Fasciola gigantica [73] and shown to inhibit mammalian cathepsin B, cathepsin $\mathrm{L}$ and other cysteine proteases, including parasite ones. In Blastocystis sp., secreted cystatin could participate in the regulation of parasitic cysteine protease activities. Cystatin can also potentially inhibit host proteases involved in MHC II antigen processing and presentation, including the key enzyme asparaginyl endopeptidase [74] and cathepsin S, the mammalian legumain [73]. 
Interestingly, a putative type I polyketide synthase (PKS) gene was also found in the Blastocystis sp. genome, potentially originating from HGT. PKS and non-ribosomal peptide synthetase (NRPS) synthesize metabolites like simple fatty acids, but also a myriad of chemical structures that possess important pharmacological activities and environmental impact, such as toxins, antibiotics or antimicrobials. Type I PKS was formerly known only from bacteria and fungi, but recently homologous genes were also discovered in some protists [75]. According to the Database for NRPS and PKS [76], the Blastocystis sp. PKS gene possesses the three essential domains, and three other domains: dehydratase, ketoacyl reductase, and enoyl reductase domains. The presence of these additional domains would permit this organism to synthesize both reduced polyketides and fatty acids. Domain comparison with other type I PKSs suggests that Blastocystis sp. PKS is similar to type I PKS from the ascomycete Cochliobolus heterostrophus, a maize pathogen that produces $\mathrm{T}$ toxin [77], a polyketide molecule that disturbs mitochondria by binding a protein of the inner mitochondrial membrane. Searching polyketide-related metabolites in the secretome of Blastocystis sp. would be of interest in order to identify molecules that could have effects on the host (Figure 3).

\section{Antioxidant system and multi drug resistance}

Like other anaerobic organisms, Blastocystis sp. has to eliminate reactive oxygen species such as superoxide anions $\left(\mathrm{O}_{2}{ }^{-}\right)$, hydrogen peroxide $\left(\mathrm{H}_{2} \mathrm{O}_{2}\right)$ and hydroxyl radicals $\left(\mathrm{HO}^{*}\right)$ resulting from metabolism. In addition, this microorganism has to cope with the oxidative burst imposed by host immune cell effectors (release of $\mathrm{O}_{2}{ }^{-}$ subsequently processed to give additional reactive oxygen species). For these reasons, to protect against oxidative injury, Blastocystis species have developed an efficient battery of antioxidant enzymes (Table S5 in Additional file 2). The first lines of defense against oxygen damage are superoxide dismutases (SODs), a family of metalloproteins catalyzing the dismutation of $\mathrm{O}_{2}{ }^{--}$to form $\mathrm{H}_{2} \mathrm{O}_{2}$ and oxygen. Genome annotation revealed the presence of two genes encoding SODs (SOD1 and SOD2) that exhibit sequence characteristics of dimeric iron-containing SODs [78] and likely protect the cytosol and MLOs, respectively, against $\mathrm{O}_{2}{ }^{\circ-}$. Catalase and ascorbate peroxidase are subsequently able to remove $\mathrm{H}_{2} \mathrm{O}_{2}$ generated by $\mathrm{SODs}$ as well as by $\mathrm{NADPH}$-dependent oxidase. However, genes encoding catalase and ascorbate peroxidase have not been identified in Blastocystis sp. nor in many unicellular parasites, including trypanosomatids and Plasmodium falciparum. Additional enzymes, glutathione peroxidase (Gpx) and thioredoxin-dependent peroxidase (commonly known as peroxyredoxin (Prx)) are able to reduce $\mathrm{H}_{2} \mathrm{O}_{2}$ to water as well as other substrates, such as hydroperoxides and peroxinitrite. In most eukaryotes, both enzymes obtain their reducing equivalents from two redox systems, the glutathione (GSH) and the thioredoxin (Trx) systems, respectively. Like P. falciparum [79], Blastocystis sp. cells possess a complete GSH synthesis pathway: the genes encoding $\gamma$-glutamylcysteine synthetase, glutathione synthetase (eu-GS group) and a functional GSH/Gpx (nonselenium Gpx belonging to the PHGpx group)/glutathione reductase system have been identified and both Gpx and glutathione reductase are probably located in the MLO. This nearly ubiquitous redox cycle is replaced by the trypanothione system in trypanosomatids [80]. Blastocystis sp. also contains genes encoding the proteins of the Trx/thioredoxin reductase (TrxR)/Prx system. Indeed, two genes encode small proteins homologous to Trx: one cytosolic and another most likely located in the MLO (Table S5 in Additional file 2). $\operatorname{Trx}$ is itself reduced by $\operatorname{TrxR}$ and three genes encoding cytosolic TrxR have been identified in Blastocystis sp. These proteins clearly belong to the high molecular weight (designated H-TrxR) group of enzymes and are similar to metazoan enzymes, including those of Homo sapiens and Drosophila melanogaster, and to those of the apicomplexan protozoa Plasmodium, Toxoplasma, and Cryptosporidium [81]. Interestingly, in contrast to apicomplexan H-TrxRs, two of the H-TrxR enzymes of Blastocystis are predicted to possess a redox active center in the carboxy-terminal domain composed of a selenocysteine (a rare amino acid encoded by the opal codon TGA, which is not recognized as a stop codon) at the penultimate position and its neighboring cysteine residue as in metazoan enzymes (selenoprotein type H-TrxR). This strongly suggests the presence of the Se-Cys insertion machinery (SECYS elements) in Blastocystis sp. Genes encoding another type of TrxR with low molecular weight (designated L-TrxR) have been identified in parasitic protozoa such as Trichomonas, Entamoeba, and Giardia but not in the genome of Blastocystis sp. These data reinforce the assumption of the exclusive occurrence of either L-TrxR or H-Trxr in genomes and of some disadvantages of possessing both types of TrxR [81]. In Blastocystis sp., at least 11 highly similar gene copies encoding predicted cytosolic Prxs have been found that clearly belong to the typical 2-Cys class of Prx. Whether sequence polymorphism of these enzymes is potentially correlated with diversified expression or even function remains to be explored. Another gene encoding a typical 2-Cys Prx, likely located in the MLO, has been identified in this parasite. Interestingly, like the homologous sequence of another stramenopile, $P$. infestans, this latter protein is fused to Trx with a WCGKC motif. As described above, Blastocystis sp. possesses a whole array of antioxidant enzymes protecting both the cytosol and 
MLO. As shown in Table S5 in Additional file 2, these enzymes have distinct phylogenetic origins and most of them probably originate from prokaryote HGT. These antioxidant proteins attract attention in unicellular parasites as they have important functions in host-parasite interactions and constitute new drug targets for the design of inhibitors. Indeed, genetic approaches have undoubtedly shown that some anti-oxidant enzymes are essential for the survival of different parasitic species [82-86].

Some genes coding for multi-drug resistance pump proteins have also been discovered in the Blastocystis sp. genome. There are two classes of multi-drug resistance genes: the first class corresponds to proteins that are energized by ATP hydrolysis; the second class includes proteins that mediate the drug efflux reaction with a proton or sodium ion gradient. Among the first class, 24 $A B C$ transporter genes were found. In eukaryotes the main physiological function of $A B C$ transporters is the export of endogenous metabolites and cytotoxic compounds [87] and eight families of $A B C$ transporters ( $\mathrm{ABC}$ A to $\mathrm{H}$ ) have been identified. The Blastocystis sp. $A B C$ transporters are included in four of these eight families (five in family A, six in family B, six in family C, three in family $\mathrm{F}$, and four not in any class). The A family is involved in lipid trafficking, and the F family in DNA repair and gene regulation. The other two families are more interesting [87], since in protozoan parasites (Leishmania spp., Trypanosoma spp., Plasmodium spp.) transporters belonging to the $\mathrm{B}$ and $\mathrm{C}$ families confer resistance to drugs. Metronidazole-resistant strains of Blastocystis sp. could have arisen through the action of these multi-drug resistance proteins (Figure 3).

\section{Conclusions}

We have provided the first genome sequence of a Blastocystis sp. subtype, which could serve in comparative genomics studies with other subtypes to provide clues to clarify how these protozoans develop pathogenicity in some humans. Analysis of this genome has revealed original traits of this lineage compared to other stramenopiles (free living and plant pathogens). Aerobic respiration has been lost, Blastocystis sp. instead having the MLO, an anaerobic organelle, which should advance our understanding of organelle evolution as the Blastocystis sp. MLO seems to be unique among organelles (Figure 2) but remains to be biochemically characterized. Some genes may have been gained through HGT, which may participate in essential functions for an intestinal parasite (adhesion, energy production). These genes probably have facilitated adaptation to intestinal environments. The Blastocystis sp. secretome has been predicted and this has permitted the identification of candidate proteins that could degrade host tissues in order to provide nutrients. Putative secretory proteins that can interfere with non-specific and specific host defense systems have also been found, enabling Blastocystis sp. to survive within this hostile environment (Figure 3). These putative secretory proteins are of particular interest as they may interact directly with host tissue and could help in understanding the host-parasite interactions and could also be used as markers to distinguish between non-pathogenic and pathogenic isolates. If their functions are essential, they could also be used to develop future vaccine formulations. The antioxidant proteins offer interesting therapeutic targets as they might be important for the parasite in fighting oxidative bursts. In summary, the deciphering of the Blastocystis sp. genome will contribute to the study of interactions between this parasite and its host at a post-genomic scale and pave the way for deciphering the host-parasite interactome. Finally, the 'Blastocystis sp. story' is reminiscent of the amoeba pathogenicity story where two morphologically indistinguishable species have different pathogenic potential [88], and this genome will help in the development of typing tools for the characterization of pathogenic isolates.

\section{Materials and methods}

\section{Genome sequencing}

The Blastocystis sp. genome was sequenced using a whole genome shotgun strategy. All data were generated by paired-end sequencing of cloned inserts using Sanger technology on ABI3730xl sequencers. Table S1 in Additional file 2 gives the number of reads obtained per library. All reads were assembled with Arachne [89]. We obtained 157 contigs that were linked into 54 supercontigs. The contig N50 was $297 \mathrm{~kb}$, and the supercontig N50 was $901 \mathrm{~kb}$ (Table S2 in Additional file 2).

\section{Genome annotation}

\section{Construction of the training set}

A set of 300 gene models from a preliminary annotation run was selected randomly, among those that were validated by Blastocystis sp. cDNAs (that is, with every intron confirmed by at least one cDNA and no exon overlapping a cDNA intron) to create a clean Blastocystis sp. training set. This training set was used to train gene prediction algorithms and optimize their parameters.

\section{Repeat masking}

Most of the genome comparisons were performed with repeat masked sequences. For this purpose, we searched and masked sequentially several kinds of repeats: known repeats and transposons available in Repbase with the Repeat Masker program [90], tandem repeats with the TRF program [91], ab initio repeat detection with RepeatScout [92], rDNA by BLATing [93] 189 rDNAs 
sequences (downloaded from GenBank), and telomeric repeats by searching 'CCCTAA' patterns in the scaffolds with the BLAST2 algorithm.

\section{GeneWise}

The UniProt [94] database was used to detect conserved genes between Blastocystis sp. and other species. As GeneWise [95] is time greedy, the UniProt database was first aligned with the Blastocystis sp. genome assembly using BLAT [93]. Subsequently, we extracted the genomic regions where no protein hit had been found by BLAT and realigned Uniprot proteins with more permissive parameters. Each significant match was then refined using GeneWise in order to identify exon/intron boundaries.

\section{GeneID and SNAP}

GeneID [96] and SNAP [97] ab initio gene prediction software were trained on 300 genes from the training set.

\section{Blastocystis sp. cDNAs}

Full-length-enriched cDNA libraries were constructed from Blastocystis sp. vacuolar forms using a SV total RNA isolation system (Promega France, Charbonnières, France) for RNA extraction. RNA quality and quantity were estimated using the Agilent bioanalyser with the RNA 6000 Nano LabChip ${ }^{\circledR}$ Kit. The clones were sequenced on the 5' end, producing 34,470 useful reads. We were able to align 33,685 cDNA sequences to the Blastocystis sp. genome assembly with the following pipeline: after masking of polyA tails, the sequences were aligned with BLAT on the assembly and all matches with scores within $99 \%$ of the best score were extended by $5 \mathrm{~kb}$ on each end, and realigned with the cDNA clones using the EST2genome software [98].

\section{Stramenopile ESTs}

A collection of 410,069 public mRNAs from the stramenopile clade $(276,208$ downloaded from the National Center for Biotechnology Information plus 43,932 and 80,929 ESTs downloaded from the Joint Genome Institute for diatoms and Ectocarpus, respectively) were first aligned with the Blastocystis sp. genome assembly using BLAT [93]. To refine BLAT alignment, we used EST2genome [98]. Each significant match was chosen for an alignment with EST2genome. BLAT alignments were made using default parameters between translated genomic and translated ESTs.

\section{Integration of resources using GAZE}

All the resources described here were used to automatically build Blastocystis sp. gene models using GAZE [99]. Individual predictions from each of the programs (that is, GeneID, SNAP, GeneWise, EST2genome) were broken down into segments (coding, intron, intergenic) and signals (start codon, stop codon, splice acceptor, splice donor, transcript start, transcript stop).
Exons predicted by ab initio software (that is, GeneWise and EST2genome) were used as coding segments. Introns predicted by GeneWise and EST2genome were used as intron segments. Intergenic segments were created from the span of each mRNA using a negative score (coercing GAZE not to split genes). Predicted repeats were used as intron and intergenic segments to avoid prediction of genes coding proteins in such regions.

The whole genome was scanned to find signals (splice sites and start and stop codons). Additionally, transcript stop signals were extracted from the ends of mRNAs (polyA tail positions).

Each segment extracted from software output that predicts exon boundaries (like GeneWise, Exonerate or $a b$ initio predictors) was used by GAZE only if GAZE chose the same boundaries. Each segment or signal from a given program was given a value reflecting our confidence in the data, and these values were used as scores for the arcs of the GAZE automaton. All signals were given a fixed score, but segment scores were context sensitive: coding segment scores were linked to the percentage identity of the alignment; intronic segment scores were linked to the percentage identity of the flanking exons. A weight was assigned to each resource to further reflect its reliability and accuracy in predicting gene models. This weight acts as a multiplier for the score of each information source, before processing by GAZE. When applied to the entire assembled sequence, GAZE predicted 4,798 gene models. Since the resource of expressed sequences in stramenopiles is limited, and some gene-free 'holes' appeared in gene-dense regions, we suspected that some genes had been missed by the annotation pipeline because of a lack of support.

\section{Additional gene models}

With the assumption that not all genes in Blastocystis sp. have EST support, we developed the following strategy to recuperate additional gene models. Ab initio (SNAP and GeneID) predictions that did not overlap GAZE gene models were selected and aligned to UniProt sequences. Predictions that had significant hits (coverage $\geq 90 \%$; e-value $\leq 10^{-5}$ ) were tagged as potential coding genes and randomly chosen genes were successfully verified by RT-PCR using the Access RT-PCR system (Promega France, Charbonnières, France). The final proteome composed of 6,020 gene models was obtained by adding 1,222 supplementary models to the 4,798 genes from the first GAZE output.

\section{Identification of orthologous genes}

We identified orthologous genes with three species: Cyanidioschyzon merolae [100], P. sojae [49] and T. pseudonana [101]. Each pair of predicted genes was aligned with the Smith-Waterman algorithm, and alignments with a score 
higher than $300($ BLOSUM62, gapo $=10$, gape $=1)$ were retained. Orthologs were defined as BRHs, that is, two genes, A from genome GA and B from genome GB, were considered orthologs if $B$ is the best match for gene $A$ in $\mathrm{GB}$ and $\mathrm{A}$ is the best match for $\mathrm{B}$ in GA.

\section{Identification of paralogous genes and duplicated blocks}

An all-against-all comparison of Blastocystis sp. proteins was performed using the Smith-Waterman algorithm implemented in the Biofacet package [102]. BRHs were identified as follows: two genes, $\mathrm{A}$ and $\mathrm{B}$, are the $\mathrm{BRH}$ if $\mathrm{B}$ is the best match for gene $\mathrm{A}$ and $\mathrm{A}$ for gene $\mathrm{B}$. The distribution of percentage identities among the pairs of BRHs is displayed in Figure S7 in Additional file 1. The distribution is widespread except for the abundant class of genes sharing $\geq 90 \%$ of identity, which represents $48 \%$ of all pairs of paralogs. We investigated this apparently recent gene duplication by selecting all pairs of genes sharing $\geq 90 \%$ identity over $\geq 50 \%$ of the length of the shortest protein (not only BRHs), which gave 1,917 gene pairs corresponding to 1,141 genes scattered in 404 gene families (19\% of Blastocystis sp. genes). The number of counterparts per gene is displayed in Figure S2 in Additional file 1. Additionally, blocks of paralogous genes, or so-called duplicated blocks, were identified by clustering the 1,917 gene pairs. The clustering was performed by single linkage clustering using the Euclidian distance between genes, and independently of gene orientation. Those distances were calculated with the gene index on each scaffold rather than the genomic position, including only the genes with paralogs. The minimal distance between two paralogous genes was set to 5 and the minimal number of genes in a cluster was set to 4 (two pairs of paralogous genes; Figure S8 in Additional file 1).

\section{Identification of candidate horizontal gene transfers}

Blastocystis sp. proteins were blasted [103] (blastx) against the protein $\mathrm{nr}$ database with the parameters '-f 100 -X 100 -e 0.00001 -E 2 -W 5', and the best hits were retained using the following criteria: for BLAST scores greater than 200, all hits with a score greater than $90 \%$ of the best score were retained; and for BLAST scores lower or equal to 200 , all hits with a score greater than $80 \%$ of the best score were retained. Then, the proteins with all their best hits in bacteria or archaea were retained as candidates that had potentially arisen from HGT. Other criteria for the blastx comparison were tested (such as $\mathrm{W}=3$ ) but we observed no significant difference in the results after the subsequent filters. Candidates with some of their best hits in stramenopiles in addition to bacteria were also retained since some HGTs may be shared between stramenopiles, and genes for which orthologs were identified in non-stramenopile species were discarded. The evolutionary origin of the candidate genes was then investigated using phylogenetic approaches (Figure S6 in Additional file 1). For each gene, homologues were retrieved from the protein $\mathrm{nr}$ database using Blastp (default parameters, except for the max-target-sequences threshold, which was fixed at 500). The sequences were aligned using Muscle 3.6 [104] (default parameters). The resulting alignments were visually inspected and manually refined using the MUST software [105]. Ambiguously aligned regions were removed prior to phylogenetic analysis.

Maximum likelihood phylogenetic tree reconstructions were carried out on the remaining positions using PhyML [106] with the Le and Gascuel (LG) model [106] with a gamma correction (four discrete classes, an estimated alpha parameter) to take into account evolutionary rate variation among sites. Tree robustness was estimated by a non-parametric bootstrap approach using PhyML and the same parameters with 100 replicates of the original dataset. Bayesian phylogenetic trees were also reconstructed using MrBayes version 3.1.2 [107]. We used a mixed model of amino acid substitution and a gamma distribution (four discrete categories plus a proportion of invariant sites) to take into account site rate variation. MrBayes was run with four chains for 1 million generations and trees were sampled every 100 generations. To construct the consensus tree, the first 1,500 trees were discarded as 'burn-in'. The candidates with clear eukaryotic origin were then discarded. This process provided 133 candidate genes (Table S3 in Additional file 2). These candidates contain a high proportion of monoexonic genes (39\%) compared to the average number of monoexonic genes in Blastocystis sp. (approximately 15\%).

\section{Protein domain analysis}

InterProScan [108] was run against all C. merolae, P. sojae, T. pseudonana and Blastocystis sp. proteins. Matches that fulfilled the following criteria were retained: match tagged as 'true positive' by InterProScan (status = $\mathrm{T}$ ); match with an e-value $\leq 10^{-1}$. A total of 2,305 InterPro domains (with IPR number) were found in Blastocystis sp., which corresponds to 4,096 proteins.

\section{Functional annotation \\ Enzyme annotation}

Enzyme detection in predicted Blastocystis sp. proteins was performed with PRIAM [109], using the PRIAM July 2006 Enzyme release. A total of 428 different EC numbers, corresponding to enzyme domains, are associated with 1,140 Blastocystis sp. proteins. Therefore, about $19 \%$ of Blastocystis sp. proteins contain at least one enzymatic domain. 
Additional file 2: Sequencing overview and assembly metric data, and the identification of horizontal gene transfer, secretory protein and antioxidant protein candidates.

Additional file 3: Proteins putatively imported in the mitochondrialike organelle. numbers using the KEGG pathway database [110]. Links between EC numbers and metabolic pathways were obtained from the KEGG website. Using this file and the PRIAM results, 906 (of the 1,140) Blastocystis sp. proteins were assigned to 201 pathways.

Identification of putative proteins imported within the MLOs

The whole proteome was scanned by two algorithms aimed at predicting proteins imported to mitochodria; MitoProt [111], which predicts mitochondrial-targeting sequences, and MitoPred [112], which predicts nuclearencoded mitochondrial proteins based on Pfam domains (animal/yeast database). After manual processing and using a script, only protein sequences with a score above 0.5 and $85 \%$ for MitoProt and MitoPred, respectively, were selected. This output file was then used in a KEGG Automatic Annotation Server (KAAS) with the bi-directional best hit method [113] in order to automatically generate KEGG pathways. Because protein domain annotations did not always provide sufficient information (PRIAM July 2006 Enzyme release), a BLAST comparison against the non-redundant database was conducted.

\section{Secretome prediction using SignalP 3.0 and pSORTII}

Prediction of secreted proteins is based on the analysis of amino-terminal secretory signal sequences (SignalP 3.0) followed by the selection of proteins predicted as extracellular by pSORTII. Each of the proteins was individually submitted to SignalP 3.0 for analysis with the following parameters: organism set to eukaryotes, output short format and protein sequence truncation after the first 50 amino acids. Results of SignalP 3.0 were exported to a temporary file, and identification of signal peptides was accomplished by parsing the results of the hidden Markov model analysis conducted by SignalP 3.0. Proteins with secretory signals were retained and analyzed on the basis of possible function in host-parasite interactions. These last ones were also analyzed using PSORT II [114], and those having a best hit as 'extracellular' were selected. The SignalP threshold value for secretory signal peptide prediction was set at 0.5 as determined for previous analyses [115] and the best hit was chosen for the PSORTII analysis. The predicted secretory proteins were then annotated as functional protein families.

\section{Additional material}

Additional file 1: Genome organization of Blastocystis sp. (introns, numbers of counterparts per gene, genome structure, and so on) and phylogenetic trees illustrating horizontal gene transfer events from prokaryotic donors to Blastocystis sp. and candidate genes for endosymbiotic gene transfers of chloroplastic origin.

\section{Abbreviations}

bp: base pair; BRH: best reciprocal hit; EST: expressed sequence tag; Gpx: glutathione peroxidase; GSH: glutathione; HGT: horizontal gene transfer; KEGG: Kyoto Encyclopedia of Genes and Genomes; MFS: major facilitator transporter; MLO: mitochondria-like organelle; NRPS: non-ribosomal peptide synthase; ORF: open reading frame; PKS: polyketide synthase; Prx: peroxyredoxin; SOD: superoxide dismutase; TCA: tricarboxylic acid; Trx: thioredoxin; TrxR: thioredoxin reductase; WGD: whole genome duplication.

\section{Acknowledgements}

We would like to thank François Enault (Université Blaise Pascal) for SignalP 3.0 analysis, David G Biron (Université Blaise Pascal) and Susan Cure (Genoscope, Evry) for manuscript reading, comments and English corrections.

\section{Author details}

${ }^{1}$ Genoscope (CEA) and CNRS UMR 8030, Université d'Evry, 2 rue Gaston Crémieux, 91057 Evry, France. ${ }^{2}$ Clermont Université, Université Blaise Pascal, Laboratoire Microorganismes: Génome et Environnement, BP 10448, F-63000 Clermont-Ferrand, France. ${ }^{3}$ CNRS, UMR 6023, LMGE, F-63177 Aubière, France. ${ }^{4}$ Center for Infection and Immunity of Lille, Institut Pasteur de Lille, F-59019 Lille Cedex, France. ${ }^{5}$ Inserm U1019, F-59000 Lille Cedex, France. ${ }^{6}$ CNRS UMR 8402, F-59021 Lille Cedex, France. 7 University Lille-Nord de France, F-59000 Lille Cedex, France. ${ }^{8}$ Laboratoire de chimie bactérienne (CNRS UPR9043), Institut de Microbiologie de la Méditerrannée, 31 chemin Joseph Aiguier, 13402 Marseille, France. ${ }^{9}$ Université de Provence, Aix-Marseille I, 3 place Victor Hugo, 13331 Marseille, France. ${ }^{10}$ Laboratory of Molecular and Cellular Parasitology, Department of Microbiology, Yong Loo Lin School of Medicine, National University of Singapore, Singapore, 5 Science Drive 2, 117597 Singapore.

\section{Authors' contributions}

FDen, PW, CPV and HEA conceived and designed the experiments. MR, IW, JP, GCN, BS, BN, CDS, AC and HEA performed the experiments. MR, IW, MD, $C T, B N, E V, C B A, F D e n, V A, F A, J M A, O J, K S W T, F D e l, P W$ and HEA analyzed the data. FDen, MR, IW, EV, CBA, FDel, CPV and HEA wrote the paper. All authors read and approved the final manuscript.

\section{Competing interests}

The authors declare that they have no competing interests.

Received: 25 October 2010 Revised: 4 January 2011

Accepted: 25 March 2011 Published: 25 March 2011

\section{References}

1. Tan KS: New insights on classification, identification, and clinical relevance of Blastocystis spp. Clin Microbiol Rev 2008, 21:639-665.

2. Alexeieff A: Sur la nature des formations dites "kystes de Trichomonas intestinalis". CR Soc Biol 1911, 71:296-298.

3. Silberman JD, Sogin ML, Leipe DD, Clark CG: Human parasite finds taxonomic home. Nature 1996, 380:398. Nakamura F, Yano TA, Hasegawa M: Phylogenetic position of Blastocystis hominis and of stramenopiles inferred from multiple molecular sequence data. J Eukaryot Microbiol 2002, 49:42-53.

5. Hoevers JD, Snowden KF: Analysis of the ITS region and partial ssu and Isu rRNA genes of Blastocystis and Proteromonas lacertae. Parasitology 2005, 131:187-196.

6. Patterson DJ: The diversity of eukaryotes. Am Nat 1999, 154:S96-S124

7. Brumpt E: Blastocystis hominis n. sp. et formes voisines. Bull Soc Pathol Exot 1912, 5:725-730
4. Arisue N, Hashimoto T, Yoshikawa H, Nakamura Y, Nakamura G, 
8. Windsor JJ, Macfarlane L, Hughes-Thapa G, Jones SK, Whiteside TM: Incidence of Blastocystis hominis in faecal samples submitted for routine microbiological analysis. Br J Biomed Sci 2002, 59:154-157.

9. Stark D, van Hal S, Marriott D, Ellis J, Harkness J: Irritable bowel syndrome: a review on the role of intestinal protozoa and the importance of their detection and diagnosis. Int J Parasitol 2007, 37:11-20.

10. Rivera WL: Phylogenetic analysis of Blastocystis isolates from animal and human hosts in the Philippines. Vet Parasitol 2008, 156:178-182.

11. Souppart L, Moussa H, Cian A, Sanciu G, Poirier P, El Alaoui H, Delbac F, Boorom K, Delhaes L, Dei-Cas E, Viscogliosi E: Subtype analysis of Blastocystis isolates from symptomatic patients in Egypt. Parasitol Res 2010, 106:505-511.

12. Marciano MG, Takizawa H, Falavigna DLM, Gomes ML: Enteroparasitosis and their ethnographic relationship to food handlers in a tourist and economic center in Paraná, southern Brazil. Rev Inst Med trop S Paulo 2009, 51:31-35.

13. Souppart L, Sanciu G, Cian A, Wawrzyniak I, Delbac F, Capron M, Dei-Cas E, Boorom K, Delhaes L, Viscogliosi E: Molecular epidemiology of human Blastocystis isolates in France. Parasitol Res 2009, 105:413-421.

14. Cirioni O, Giacometti A, Drenaggi D, Ancarani F, Scalise G: Prevalence and clinical relevance of Blastocystis hominis in diverse patient cohorts. Eur $J$ Epidemiol 1999, 15:389-393.

15. Lucia JF, Aguilar C, Betran A: Blastocystis hominis colitis in a haemophilic patient as a cause of lower gastrointestinal bleeding. Haemophilia 2007, 13:224-225.

16. Stenzel DJ, Boreham PF: Blastocystis hominis revisited. Clin Microbiol Rev 1996, 9:563-584.

17. Stensvold CR, Suresh GK, Tan KS, Thompson RC, Traub RJ, Viscogliosi E, Yoshikawa H, Clark CG: Terminology for Blastocystis subtypes - a consensus. Trends Parasitol 2007, 23:93-96.

18. Wong KH, Ng GC, Lin RT, Yoshikawa H, Taylor MB, Tan KS: Predominance of subtype 3 among Blastocystis isolates from a major hospital in Singapore. Parasitol Res 2008, 102:663-670.

19. Iguchi A, Ebisu A, Nagata S, Saitou Y, Yoshikawa H, Iwatani S, Kimata I: Infectivity of different genotypes of human Blastocystis hominis isolates in chickens and rats. Parasitol Int 2007, 56:107-112.

20. Boorom KF, Smith H, Nimri L, Viscogliosi E, Spanakos G, Parkar U, Li LH, Zhou XN, Ok UZ, Leelayoova S, Jones MS: Oh my aching gut: irritable bowel syndrome, Blastocystis, and asymptomatic infection. Parasit Vectors 2008, 1:40.

21. Zierdt CH: Blastocystis hominis-past and future. Clin Microbiol Rev 1991, 4:61-79.

22. Nasirudeen AMA, Eu-Hian Y, Singh M, Tan KSW: Metronidazole induces programmed cell death in the protozoan parasite Blastocystis hominis. Microbiology 2004, 150:33-43.

23. Stechmann A, Hamblin K, Perez-Brocal V, Gaston D, Richmond GS, van der Giezen M, Clark CG, Roger AJ: Organelles in Blastocystis that blur the distinction between mitochondria and hydrogenosomes. Curr Biol 2008, 18:580-585.

24. Wawrzyniak I, Roussel M, Diogon M, Couloux A, Texier C, Tan KS, Vivares CP, Delbac F, Wincker P, El Alaoui H: Complete circular DNA in the mitochondria-like organelles of Blastocystis hominis. Int J Parasitol 2008, 38:1377-1382.

25. Windsor JJ: Blastocystis hominis and Dientamoeba fragilis: neglected human protozoa. The Biomedical Scientist 2007, 64:524-527.

26. al-Tawil YS, Gilger MA, Gopalakrishna GS, Langston C, Bommer KE: Invasive Blastocystis hominis infection in a child. Arch Pediatr Adolesc Med 1994, 148:882-885.

27. Jaillon $\mathrm{O}$, Aury JM, Brunet F, Petit JL, Stange-Thomann N, Mauceli E, Bouneau L, Fischer C, Ozouf-Costaz C, Bernot A, Nicaud S, Jaffe D, Fisher S, Lutfalla G, Dossat C, Segurens B, Dasilva C, Salanoubat M, Levy M, Boudet N, Castellano S, Anthouard V, Jubin C, Castelli V, Katinka M, Vacherie B, Biémont C, Skalli Z, Cattolico L, Poulain J, et al: Genome duplication in the teleost fish Tetraodon nigroviridis reveals the early vertebrate protokaryotype. Nature 2004, 431:946-957.

28. Davis JC, Petrov DA: Preferential duplication of conserved proteins in eukaryotic genomes. PLoS Biol 2004, 2:E55.

29. Wolfe KH, Shields DC: Molecular evidence for an ancient duplication of the entire yeast genome. Nature 1997, 387:708-713.

30. Semple C, Wolfe KH: Gene duplication and gene conversion in the Caenorhabditis elegans genome. J Mol Evol 1999, 48:555-564.
31. Vision TJ, Brown DG, Tanksley SD: The origins of genomic duplications in Arabidopsis. Science 2000, 290:2114-2117.

32. Sankoff D: Gene and genome duplication. Curr Opin Genet Dev 2001, 11:681-684.

33. Bailey JA, Gu Z, Clark RA, Reinert K, Samonte RV, Schwartz S, Adams MD, Myers EW, Li PW, Eichler EE: Recent segmental duplications in the human genome. Science 2002, 297:1003-1007.

34. Wolfe KH, Li WH: Molecular evolution meets the genomics revolution. Nat Genet 2003, 33(Suppl):255-265.

35. Andersson JO, Roger AJ: A cyanobacterial gene in nonphotosynthetic protists - an early chloroplast acquisition in eukaryotes?. Curr Biol 2002, 12:115-119.

36. Maruyama S, Misawa K, Iseki M, Watanabe M, Nozaki H: Origins of a cyanobacterial 6-phosphogluconate dehydrogenase in plastid-lacking eukaryotes. BMC Evol Biol 2008, 8:151

37. Archibald JM: The puzzle of plastid evolution. Curr Biol 2009, 19:R81-88.

38. Keeling PJ, Palmer JD: Horizontal gene transfer in eukaryotic evolution. Nat Rev Genet 2008, 9:605-618.

39. Andersson JO: Horizontal gene transfer between microbial eukaryotes. Methods Mol Biol 2009, 532:473-487.

40. Keeling PJ: Functional and ecological impacts of horizontal gene transfer in eukaryotes. Curr Opin Genet Dev 2009, 19:613-619.

41. Bowler C, Allen AE, Badger JH, Grimwood J, Jabbari K, Kuo A, Maheswari U, Martens C, Maumus F, Otillar RP, Rayko E, Salamov A, Vandepoele K, Beszteri B, Gruber A, Heijde M, Katinka M, Mock T, Valentin K, Verret F, Berges JA, Brownlee C, Cadoret JP, Chiovitti A, Choi CJ, Coesel S, De Martino A, Detter JC, Durkin C, Falciatore A, et al: The Phaeodactylum genome reveals the evolutionary history of diatom genomes. Nature 2008, 456:239-244.

42. Richards $T A$, Dacks JB, Jenkinson JM, Thornton $C R$, Talbot NJ: Evolution of filamentous plant pathogens: gene exchange across eukaryotic kingdoms. Curr Biol 2006, 16:1857-1864.

43. Law CJ, Maloney PC, Wang DN: Ins and outs of major facilitator superfamily antiporters. Annu Rev Microbiol 2008, 62:289-305.

44. Berger P, Papazian L, Drancourt M, La Scola B, Auffray JP, Raoult D: Amebaassociated microorganisms and diagnosis of nosocomial pneumonia. Emerg Infect Dis 2006, 12:248-255.

45. Embley TM, van der Giezen M, Horner DS, Dyal PL, Foster P: Mitochondria and hydrogenosomes are two forms of the same fundamental organelle. Philos Trans R Soc Lond B Biol Sci 2003, 358:191-201, discussion 201-192.

46. Field J, Rosenthal B, Samuelson J: Early lateral transfer of genes encoding malic enzyme, acetyl-CoA synthetase and alcohol dehydrogenases from anaerobic prokaryotes to Entamoeba histolytica. Mol Microbiol 2000, 38:446-455.

47. Nixon JE, Wang A, Field J, Morrison HG, McArthur AG, Sogin ML, Loftus BJ, Samuelson J: Evidence for lateral transfer of genes encoding ferredoxins, nitroreductases, NADH oxidase, and alcohol dehydrogenase 3 from anaerobic prokaryotes to Giardia lamblia and Entamoeba histolytica. Eukaryot Cell 2002, 1:181-190.

48. Martin FN, Bensasson D, Tyler BM, Boore JL: Mitochondrial genome sequences and comparative genomics of Phytophthora ramorum and $P$. sojae. Curr Genet 2007, 51:285-296.

49. Perez-Brocal V, Clark CG: Analysis of two genomes from the mitochondrion-like organelle of the intestinal parasite Blastocystis: complete sequences, gene content, and genome organization. Mol Biol Evol 2008, 25:2475-2482.

50. Chacinska A, Koehler CM, Milenkovic D, Lithgow T, Pfanner N: Importing mitochondrial proteins: machineries and mechanisms. Cell 2009, 138:628-644.

51. Hoffmeister M, van der Klei A, Rotte C, van Grinsven KW, van Hellemond JJ, Henze K, Tielens AG, Martin W: Euglena gracilis rhodoquinone:ubiquinone ratio and mitochondrial proteome differ under aerobic and anaerobic conditions. J Biol Chem 2004, 279:22422-22429.

52. Tielens AG, Rotte C, van Hellemond JJ, Martin W: Mitochondria as we don't know them. Trends Biochem Sci 2002, 27:564-572.

53. Putignani L, Tait A, Smith HV, Horner D, Tovar J, Tetley L, Wastling JM: Characterization of a mitochondrion-like organelle in Cryptosporidium parvum. Parasitology 2004, 129:1-18.

54. Henriquez FL, Richards TA, Roberts F, McLeod R, Roberts CW: The unusual mitochondrial compartment of Cryptosporidium parvum. Trends Parasitol 2005, 21:68-74. 
55. Lill R: Function and biogenesis of iron-sulphur proteins. Nature 2009, 460:831-838.

56. Hiltunen JK, Schonauer MS, Autio KJ, Mittelmeier TM, Kastaniotis AJ, Dieckmann CL: Mitochondrial fatty acid synthesis type II: more than just fatty acids. J Biol Chem 2009, 284:9011-9015.

57. Corrales RM, Sereno D, Mathieu-Daude F: Deciphering the Leishmania exoproteome: what we know and what we can learn. FEMS Immunol Med Microbiol 2010, 58:27-38.

58. Bell A, Monaghan P, Page AP: Peptidyl-prolyl cis-trans isomerases (immunophilins) and their roles in parasite biochemistry, host-parasite interaction and antiparasitic drug action. Int J Parasitol 2006, 36:261-276.

59. Henderson B: Cell stress proteins as modulators of bacteria - host interactions. Novartis Found Symp 2008, 291:141-154, discussion 154-149, 221-144.

60. Golding H, Aliberti J, King LR, Manischewitz J, Andersen J, Valenzuela J, Landau NR, Sher A: Inhibition of HIV-1 infection by a CCR5-binding cyclophilin from Toxoplasma gondii. Blood 2003, 102:3280-3286.

61. Klion AD, Donelson JE: OvGalBP, a filarial antigen with homology to vertebrate galactoside-binding proteins. Mol Biochem Parasitol 1994, 65:305-315.

62. Toscano MA, Commodaro AG, llarregui JM, Bianco GA, Liberman A, Serra HM, Hirabayashi J, Rizzo LV, Rabinovich GA: Galectin-1 suppresses autoimmune retinal disease by promoting concomitant Th2- and T regulatory-mediated anti-inflammatory responses. J Immunol 2006, 176:6323-6332.

63. Katoh S, Ishii N, Nobumoto A, Takeshita K, Dai SY, Shinonaga R, Niki T, Nishi N, Tominaga A, Yamauchi A, Hirashima M: Galectin-9 inhibits CD44hyaluronan interaction and suppresses a murine model of allergic asthma. Am J Respir Crit Care Med 2007, 176:27-35.

64. Kubach J, Lutter P, Bopp T, Stoll S, Becker C, Huter E, Richter C, Weingarten $P$, Warger T, Knop J, Müllner S, Wijdenes J, Schild H, Schmitt E, Jonuleit H: Human CD4+CD25+ regulatory T cells: proteome analysis identifies galectin-10 as a novel marker essential for their anergy and suppressive function. Blood 2007, 110:1550-1558.

65. McKerrow JH, Sun E, Rosenthal PJ, Bouvier J: The proteases and pathogenicity of parasitic protozoa. Annu Rev Microbiol 1993, 47:821-853.

66. Klemba M, Goldberg DE: Biological roles of proteases in parasitic protozoa. Annu Rev Biochem 2002, 71:275-305.

67. Puthia MK, Vaithilingam A, Lu J, Tan KS: Degradation of human secretory immunoglobulin A by Blastocystis. Parasitol Res 2005, 97:386-389

68. Dalton JP, Brindley PJ, Donnelly S, Robinson MW: The enigmatic asparaginyl endopeptidase of helminth parasites. Trends Parasitol 2009, 25:59-61.

69. Wu B, Yin J, Texier C, Roussel M, Tan KS: Blastocystis legumain is localized on the cell surface, and specific inhibition of its activity implicates a prosurvival role for the enzyme. J Biol Chem 2010, 285:1790-1798.

70. Morita $Y$, Araki H, Sugimoto T, Takeuchi K, Yamane T, Maeda T, Yamamoto Y, Nishi K, Asano M, Shirahama-Noda K, Nishimura M, Uzu T, Hara-Nishimura I, Koya D, Kashiwagi A, Ohkubo I: Legumain/asparaginyl endopeptidase controls extracellular matrix remodeling through the degradation of fibronectin in mouse renal proximal tubular cells. FEBS Lett 2007, 581:1417-1424.

71. Hartmann S, Lucius R: Modulation of host immune responses by nematode cystatins. Int J Parasitol 2003, 33:1291-1302.

72. Martzen MR, Peanasky RJ: Ascaris suum: biosynthesis and isoinhibitor profile of chymotrypsin/elastase isoinhibitors. Exp Parasitol 1985, 59:313-320.

73. Tarasuk M, Vichasri Grams S, Viyanant V, Grams R: Type I cystatin (stefin) is a major component of Fasciola gigantica excretion/secretion product. Mol Biochem Parasitol 2009, 167:60-71

74. Manoury B, Gregory WF, Maizels RM, Watts C: Bm-CPI-2, a cystatin homolog secreted by the filarial parasite Brugia malayi, inhibits class II MHC-restricted antigen processing. Curr Biol 2001, 11:447-451.

75. Smith S, Tsai SC: The type I fatty acid and polyketide synthases: a tale of two megasynthases. Nat Prod Rep 2007, 24:1041-1072.

76. Database for NRPS and PKS. [http://www.nii.res.in/searchall.html].

77. Yang G, Rose MS, Turgeon BG, Yoder OC: A polyketide synthase is required for fungal virulence and production of the polyketide T-toxin. Plant Cell 1996, 8:2139-2150.

78. Wintjens R, Noel C, May AC, Gerbod D, Dufernez F, Capron M, Viscogliosi E, Rooman M: Specificity and phenetic relationships of iron- and manganese-containing superoxide dismutases on the basis of structure and sequence comparisons. J Biol Chem 2004, 279:9248-9254.

79. Muller S: Redox and antioxidant systems of the malaria parasite Plasmodium falciparum. Mol Microbiol 2004, 53:1291-1305.

80. Muller S, Liebau E, Walter RD, Krauth-Siegel RL: Thiol-based redox metabolism of protozoan parasites. Trends Parasitol 2003, 19:320-328.

81. Hirt RP, Muller S, Embley TM, Coombs GH: The diversity and evolution of thioredoxin reductase: new perspectives. Trends Parasitol 2002, 18:302-308.

82. Krnajski Z, Gilberger TW, Walter RD, Cowman AF, Muller S: Thioredoxin reductase is essential for the survival of Plasmodium falciparum erythrocytic stages. J Biol Chem 2002, 277:25970-25975.

83. Krieger S, Schwarz W, Ariyanayagam MR, Fairlamb AH, Krauth-Siegel RL, Clayton C: Trypanosomes lacking trypanothione reductase are avirulent and show increased sensitivity to oxidative stress. Mol Microbio/ 2000, 35:542-552.

84. Plewes KA, Barr SD, Gedamu L: Iron superoxide dismutases targeted to the glycosomes of Leishmania chagasi are important for survival. Infect Immun 2003, 71:5910-5920.

85. Wilkinson SR, Horn D, Prathalingam SR, Kelly JM: RNA interference identifies two hydroperoxide metabolizing enzymes that are essential to the bloodstream form of the african trypanosome. J Biol Chem 2003, 278:31640-31646.

86. Piacenza L, Alvarez MN, Peluffo G, Radi R: Fighting the oxidative assault: the Trypanosoma cruzi journey to infection. Curr Opin Microbiol 2009, 12:415-421.

87. Sauvage $V$, Aubert $D$, Escotte-Binet $S$, Villena I: The role of ATP-binding cassette $(A B C)$ proteins in protozoan parasites. Mol Biochem Parasitol 2009, 167:81-94.

88. Pinilla AE, Lopez MC, Viasus DF: [History of the Entamoeba histolytica protozoan]. Rev Med Chil 2008, 136:118-124

89. Jaffe DB, Butler J, Gnerre S, Mauceli E, Lindblad-Toh K, Mesirov JP, Zody MC Lander ES: Whole-genome sequence assembly for mammalian genomes: Arachne 2. Genome Res 2003, 13:91-96.

90. Chen N: Using RepeatMasker to identify repetitive elements in genomic sequences. Curr Protoc Bioinformatics 2004, Chapter 4, Unit 410.

91. Benson G: Tandem repeats finder: a program to analyze DNA sequences. Nucleic Acids Res 1999, 27:573-580.

92. Price $A L$, Jones NC, Pevzner PA: De novo identification of repeat families in large genomes. Bioinformatics 2005, 21(Suppl 1):i351-358.

93. Kent WJ: BLAT - the BLAST-like alignment tool. Genome Res 2002, 12:656-664.

94. Bairoch A, Apweiler R, Wu CH, Barker WC, Boeckmann B, Ferro S, Gasteiger E, Huang H, Lopez R, Magrane M, Martin MJ, Natale DA, O'Donovan C, Redaschi N, Yeh LS: The Universal Protein Resource (UniProt). Nucleic Acids Res 2005, 33:D154-159.

95. Birney E, Durbin R: Using GeneWise in the Drosophila annotation experiment. Genome Res 2000, 10:547-548.

96. Parra G, Blanco E, Guigo R: GenelD in Drosophila. Genome Res 2000 10:511-515.

97. Korf I: Gene finding in novel genomes. BMC Bioinformatics 2004, 5:59.

98. Mott R: EST_GENOME: a program to align spliced DNA sequences to unspliced genomic DNA. Comput Appl Biosci 1997, 13:477-478.

99. Howe KL, Chothia T, Durbin R: GAZE: a generic framework for the integration of gene-prediction data by dynamic programming. Genome Res 2002, 12:1418-1427.

100. Nozaki H, Takano H, Misumi O, Terasawa K, Matsuzaki M, Maruyama S, Nishida K, Yagisawa F, Yoshida Y, Fujiwara T, Takio S, Tamura K, Chung SJ, Nakamura S, Kuroiwa H, Tanaka K, Sato N, Kuroiwa T: A 100\%-complete sequence reveals unusually simple genomic features in the hot-spring red alga Cyanidioschyzon merolae. BMC Biol 2007, 5:28.

101. Armbrust EV, Berges JA, Bowler C, Green BR, Martinez D, Putnam NH, Zhou S, Allen AE, Apt KE, Bechner M, Brzezinski MA, Chaal BK, Chiovitti A, Davis AK, Demarest MS, Detter JC, Glavina T, Goodstein D, Hadi MZ, Hellsten U, Hildebrand M, Jenkins BD, Jurka J, Kapitonov W, Kröger N, Lau WW, Lane TW, Larimer FW, Lippmeier JC, Lucas S, et al: The genome of the diatom Thalassiosira pseudonana: ecology, evolution, and metabolism. Science 2004, 306:79-86.

102. GenomeQuest. [http://www.gene-it.com].

103. Altschul SF, Gish W, Miller W, Myers EW, Lipman DJ: Basic local alignment search tool. J Mol Biol 1990, 215:403-410. 
104. Edgar RC: MUSCLE: a multiple sequence alignment method with reduced time and space complexity. BMC Bioinformatics 2004, 5:113.

105. Philippe H: MUST, a computer package of Management Utilities for Sequences and Trees. Nucleic Acids Res 1993, 21:5264-5272.

106. Guindon S, Dufayard JF, Lefort V, Anisimova M, Hordijk W, Gascuel O: New algorithms and methods to estimate maximum-likelihood phylogenies: assessing the performance of PhyML 3.0. Syst Biol 2010, 59:307-321.

107. Ronquist F, Huelsenbeck JP: MrBayes 3: Bayesian phylogenetic inference under mixed models. Bioinformatics 2003, 19:1572-1574.

108. Zdobnov EM, Apweiler R: InterProScan - an integration platform for the signature-recognition methods in InterPro. Bioinformatics 2001, 17:847-848.

109. Claudel-Renard C, Chevalet C, Faraut T, Kahn D: Enzyme-specific profiles for genome annotation: PRIAM. Nucleic Acids Res 2003, 31:6633-6639.

110. Kanehisa M, Goto S, Kawashima S, Nakaya A: The KEGG databases at GenomeNet. Nucleic Acids Res 2002, 30:42-46.

111. MITOPROT. [http://ihg2.helmholtz-muenchen.de/ihg/mitoprot.html].

112. MITOPRED. [http://bioapps.rit.albany.edu/MITOPRED/]

113. KEGG Automatic Annotation Server. [http://www.genome.jp/kaas-bin/ kaas_main].

114. PSORT II Prediction. [http://psort.ims.u-tokyo.ac.jp/form2.html].

115. Nagaraj SH, Gasser RB, Ranganathan S: Needles in the EST haystack: largescale identification and analysis of excretory-secretory (ES) proteins in parasitic nematodes using expressed sequence tags (ESTs). PLoS Negl Trop Dis 2008, 2:e301.

doi:10.1186/gb-2011-12-3-r29

Cite this article as: Denoeud et al.: Genome sequence of the

stramenopile Blastocystis, a human anaerobic parasite. Genome Biology 2011 12:R29.

\section{Submit your next manuscript to BioMed Central} and take full advantage of:

- Convenient online submission

- Thorough peer review

- No space constraints or color figure charges

- Immediate publication on acceptance

- Inclusion in PubMed, CAS, Scopus and Google Scholar

- Research which is freely available for redistribution

Submit your manuscript at www.biomedcentral.com/submit 